\title{
Robust Fault Diagnosis Algorithm of Nonlinear System based on Group Algebra Theory
}

\author{
Zhao Jing-yun ${ }^{1}$, Han Xiao $^{1}$, Fu Zhen-duo ${ }^{2}$ and Liao Yue-feng ${ }^{1}$ \\ 1. Henan Mechanical and Electrical Engineering, Xinxiang 453002, China \\ 2. College of Automation, Harbin Engineering University, Harbin 150001, China, \\ fuzhenduo@126.com
}

\begin{abstract}
Dealt with the Lipschitz nonlinear system with unknown input disturbances, the robust fault diagnosis observer algorithm is proposed. In order to improve the limitations of the traditional methods on assumption that the norm of unknown input disturbances is bounded, the disturbances constraint condition that satisfies the system stability is derived via the group algebra theory. It is verified that a class of systems under the norm bounded constraint condition is unstable. The robustness performance index is defined to ensure the robustness of the fault diagnosis observer designed. The fault diagnosis observer constructed is robust asymptotic convergence observer under the constraint of robustness performance index derived using the Gronwall lemma. In the decision-making, adaptive threshold is designed. Finally, the simulations are performed on missile attitude control system. Simulation results show that the effectiveness of the algorithm proposed.
\end{abstract}

Keywords: Fault diagnosis; Attitude control system; Robustness; Nonlinear

\section{Introduction}

With the development of the modern defense technology, it makes the missile toward a wide range of high mobility and high Mach direction. But missile systems also appear strong nonlinear coupling characteristics based on high performance index. But there are few fault diagnosis methods for missile control systems based on analytical model [1-3]. Usually, missile motions decompose into longitudinal motion, lateral motion and rolling motion [4-6]. The longitudinal motion can exist independently, but the lateral motion exists with the longitudinal motion simultaneously. In this paper, the fault diagnosis algorithm is proposed for the missile longitudinal motion control system.

The robust fault diagnosis observer is designed based on unknown input observer theory in reference [7], and state estimation errors decouple from disturbances. However, the effect of disturbances to the system is not studied deeply. The linear system model and the algorithm presume that the disturbances distribution matrix is known restrict the algorithm application range.

Dealt with the sensor fault and actuators fault in F-16, the EKF fault diagnosis filter is designed in [8]. Simulations show the well results, but the linearization from the nonlinear system affects the diagnosis accuracy. Robust fault diagnosis observer is designed for nonlinear system and then the system residuals are constructed by LMIs in [9], but the norm bound restriction condition and its impact on the system stability is worth to study in [9].

In order to improve the deficiency mentioned above, the uncertainty of system model, unknown input disturbances and the system nonlinear characteristic are considered abundantly in this paper. Dealt with the deficiency [10-13] on assumption that the disturbance is norm bound, a novel constraint condition for disturbance is proposed. The robustness performance index is defined to assure the algorithm robustness. And constraint condition of the robustness performance index is proposed by Gronwall and the 
condition assures that the robust fault diagnosis observer proposed is asymptotic convergence. Furthermore, the threshold is designed in fault decision section. The deficiency is avoided compared to traditional fault decision methods [14-17].

\section{Problem Statement}

Consider the system uncertainty and unknown input disturbances, system state space model:

$$
\begin{aligned}
& \dot{\boldsymbol{x}}(t)=(\boldsymbol{A}+\Delta \boldsymbol{A}) \boldsymbol{x}(t)+(\boldsymbol{B}+\Delta \boldsymbol{B}) \boldsymbol{u}(t)+\boldsymbol{h}(\boldsymbol{x}(t), \boldsymbol{u}(t))+\boldsymbol{g}_{0}(\boldsymbol{x}(t), \boldsymbol{u}(t), \boldsymbol{d}(t), t)+\boldsymbol{f}(t) \\
& \boldsymbol{y}(t)=\boldsymbol{C} \boldsymbol{x}(t)
\end{aligned}
$$

where, $\boldsymbol{E}_{1}, \boldsymbol{E}_{2}, \boldsymbol{F}_{1}, \boldsymbol{F}_{2}$ are known matrix. $\Delta \boldsymbol{A}$ and $\Delta \boldsymbol{B}$ are model errors as follows:

$$
\left\{\begin{array}{l}
\boldsymbol{\Omega}_{1}=\left\{\Delta \boldsymbol{A} \mid \Delta \boldsymbol{A}=\boldsymbol{E}_{1} \Sigma_{1} \boldsymbol{F}_{1}, \quad \Sigma_{1}^{T} \Sigma_{1} \leq \boldsymbol{I}\right\} \\
\boldsymbol{\Omega}_{2}=\left\{\Delta \boldsymbol{B} \mid \Delta \boldsymbol{B}=\boldsymbol{E}_{2} \boldsymbol{\Sigma}_{2} \boldsymbol{F}_{2}, \quad \Sigma_{2}^{T} \Sigma_{2} \leq \boldsymbol{I}\right\}
\end{array}\right.
$$

Therefore, system (1) can be rewritten as:

$$
\dot{\boldsymbol{x}}(t)=\boldsymbol{A} \boldsymbol{x}(t)+\boldsymbol{B u}(t)+\boldsymbol{h}(\boldsymbol{x}(t), \boldsymbol{u}(t))+\boldsymbol{g}(\boldsymbol{x}(t), \boldsymbol{u}(t), \boldsymbol{d}(t), t)+\boldsymbol{f}(t)
$$

where, $\boldsymbol{x}(0)=\boldsymbol{x}_{0}$ is system state initial value, $\boldsymbol{x}(t) \in \boldsymbol{R}^{n}$ is system state, $\boldsymbol{u}(t) \in \boldsymbol{R}^{p}$ is control input, $\boldsymbol{y}(t) \in \boldsymbol{R}^{q}$ is system output, $\boldsymbol{g} \in \boldsymbol{R}^{n}$ is unknown input including disturbances and system noise, nonlinear function $\boldsymbol{g}$ and $\boldsymbol{h}$ are continuous and differential, $\boldsymbol{f}(t) \in \boldsymbol{R}^{n}$ is system fault. $\boldsymbol{A}, \boldsymbol{B}$ and $\boldsymbol{C}$ are known matrix. The missile longitudinal motion is as figure 1:

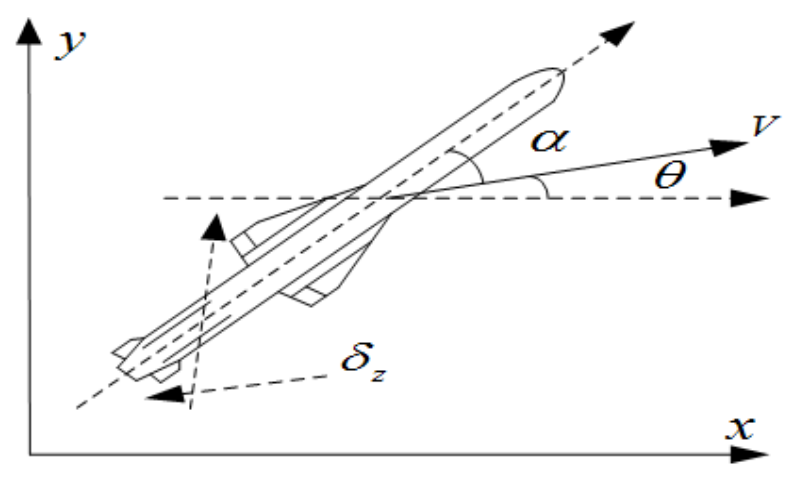

Figure 1. Missile Longitudinal Motion

Without loss of generality, assumptions are given for (2)-(3) as follows:

(A1) $(\boldsymbol{C}, \boldsymbol{A})$ are observable, and $\boldsymbol{A}$ is Hurwitz matrix;

(A2) The supreme of disturbance is defined as: $\lambda_{0}=\sup _{t \in[0, T]}\|\boldsymbol{g}(\boldsymbol{x}(t), \boldsymbol{u}(t), \boldsymbol{d}(t), t)\|$

It is noted that the input disturbances are defined as norm bound in most of fault diagnosis algorithms (as A2), for example: $\sup _{t \in[0, T]}\|\boldsymbol{g}(\boldsymbol{x}(t), \boldsymbol{u}(t), \boldsymbol{d}(t), t)\|=\lambda_{0}=5$

But the constraint condition for the external disturbances is worth to study. Limitations of A2 will be explained in section 3. There exist Lipschitz constant $\lambda_{1}$ :

$$
\|\boldsymbol{h}(\boldsymbol{x}(t), \boldsymbol{u}(t))-\boldsymbol{h}(\hat{\boldsymbol{x}}(t), \boldsymbol{u}(t))\| \leq \lambda_{1}\|\boldsymbol{x}(t)-\hat{\boldsymbol{x}}(t)\|
$$

Bounded linear operator semi-group [18]: Set $X$ as the Banach space, $\{T(t), t \geq 0\}$ represents the bounded linear operator family of $\mathrm{X} \rightarrow \mathrm{X} .\{T(t), t \geq 0\}$ is called bounded linear operator semi-group when it satisfies the following conditions:

(1) $T(0)=\boldsymbol{I}$

(2) $T(t+s)=T(t) T(s)=T(s) T(t) \quad(t, s \geq 0)$ 
(3) $\lim _{t \rightarrow 0^{+}}\|T(t) x-x\|=0, x \in X$

Gronwall Lemma [19]: There exists positive semi-define continuous function $\partial_{1}(t), \partial_{2}(t), \partial_{3}(t)$ and $\partial_{0} \in R^{+}$such that the following formulation holds:

$$
\partial_{1}(t) \leq \partial_{0}+\int_{0}^{t}\left[\partial_{2}(\tau) \partial_{1}(\tau)+\partial_{3}(\tau)\right] d \tau
$$

And then, $\partial_{1}(t) \leq \partial_{0} \exp \left[\int_{0}^{t}\left[\partial_{2}(\tau)+\partial_{3}(\tau) / \partial_{0}\right] d \tau\right]$

The system state-space model can be obtained by Gronwall lemma. Considering the complexity and particularity of missile control system, in the next two sections we perform the fault diagnosis algorithm from two aspects: stability and robust of the observer proposed.

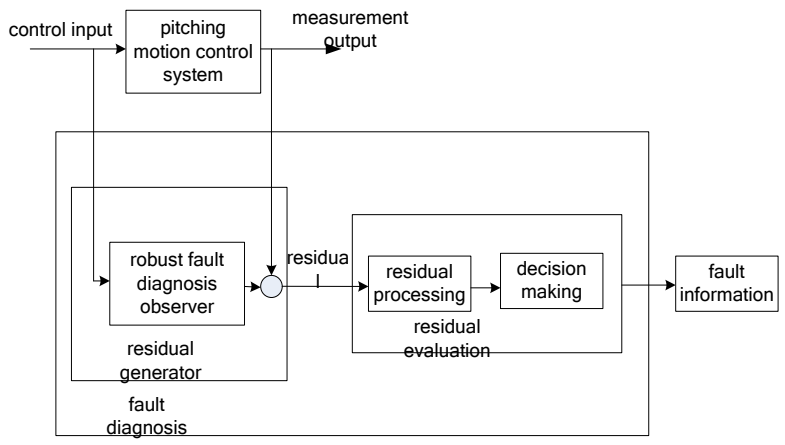

\section{Figure 2. Schematic of the Fault Diagnosis Procedures}

Most of related papers assume that the constraint condition of external disturbances are norm bounded like A2, however, a kind of nonlinear systems are unstable under the hypothetical constraint condition. Dealt with the limitations of disturbances constraint condition mentioned, a novel constraint condition of external disturbances is derived in section 3, the systems hold stable under the condition proposed. Furthermore, in order to ensure robustness of fault diagnosis algorithm proposed to external disturbances, robustness performance index is defined in section 4. The schematic of fault diagnosis algorithm this paper is depicted in Fig.2.

\section{Stability Analysis of Disturbances System}

Because of the modeling errors and external disturbances interference, the ideal statespace description can not be modeled perfectly. The unknown input or external disturbances are added to system for matching the model uncertainties. Therefore, the effect of external disturbances act on the system is considerable to research. In process of missile control system design, it is inappropriate to simply assume that external disturbances are norm bounded. The constraint condition of external disturbances proposed in this section is suitable for prior period analysis of missile control system design.

Theorem 1: There exists $M \geq 1, \omega<0, t \geq 0$ to make nonlinear system (2)-(3) with external disturbances hold stable. When the constraint condition of external disturbances is:

$$
\|\boldsymbol{g}(\boldsymbol{x}(t), \boldsymbol{u}(t), \boldsymbol{d}(t), t)\|<\left(\beta_{1}(t)-\beta_{0}(t)\right)-\omega\|\boldsymbol{x}(\tau)\| / M
$$

with definition: $\beta_{0}(t)=\|\boldsymbol{B} \boldsymbol{u}(t)\|, \beta_{1}(t)=\|\boldsymbol{h}(\boldsymbol{x}(t), \boldsymbol{u}(t))\|$.

Proof: The system matrix $\boldsymbol{A}$ is Hurwitz matrix from the assumption (A1), therefore, the matrix $\boldsymbol{A}$ can form an operator set for purpose of generating asymptotic convergence linear semi-group $\zeta_{t}$. And then, there exists $M \geq 1, \omega<0, t \geq 0$ such that the following inequality holds: 


$$
\left\|\zeta_{t}\right\| \leq M \exp (\omega t)
$$

In the fault-free mode, the state-space description of system (2)-(3) with external disturbances is:

$$
\boldsymbol{x}(t)=\boldsymbol{x}(0)+\int_{0}^{t}[\boldsymbol{A x}(\tau)+\boldsymbol{B} \boldsymbol{u}(\tau)+\boldsymbol{h}(\boldsymbol{x}(\tau), \boldsymbol{u}(\tau))+\boldsymbol{g}(\boldsymbol{x}(\tau), \boldsymbol{u}(\tau), \boldsymbol{d}(\tau), \tau)] d \tau
$$

Therefore, $\exists$ stable linear semi-group $\zeta_{t}$ make (6) hold:

$$
\boldsymbol{x}(t)=\zeta_{t} \boldsymbol{x}(0)+\zeta_{t-\tau} \int_{0}^{t}[\boldsymbol{B} \boldsymbol{u}(\tau)+\boldsymbol{h}(\boldsymbol{x}(\tau), \boldsymbol{u}(\tau))+\boldsymbol{g}(\boldsymbol{x}(\tau), \boldsymbol{u}(\tau), \boldsymbol{d}(\tau), \tau)] d \tau
$$

Apply the 2-norm to formula (6):

$$
\|\boldsymbol{x}(t)\|=\left\|\zeta_{t} \boldsymbol{x}(0)+\zeta_{t-\tau} \int_{0}^{t}[\boldsymbol{B u}(\tau)+\boldsymbol{h}(\boldsymbol{x}(\tau), \boldsymbol{u}(\tau))+\boldsymbol{g}(\boldsymbol{x}(\tau), \boldsymbol{u}(\tau), \boldsymbol{d}(\tau), \tau)] d \tau\right\|
$$

The simplification form of formula (7) is:

$$
\Xi=\boldsymbol{B u}(\tau)+\boldsymbol{h}(\boldsymbol{x}(\tau), \boldsymbol{u}(\tau))+\boldsymbol{g}(\boldsymbol{x}(\tau), \boldsymbol{u}(\tau), \boldsymbol{d}(\tau), \tau)
$$

Initial value of state is $\boldsymbol{x}_{0}=\boldsymbol{x}(0)$, we set $a=\|\boldsymbol{x}(0)\|$. From constraint of linear semi-group $\zeta_{t}$ in formula (4) and norm basic principle, the inequality constraint condition of formula (7) should be satisfied as following:

$$
\|\boldsymbol{x}(t)\| \leq M a \exp (\omega t)+\int_{0}^{t}\|M \exp [\omega(t-\tau)] \Xi\| d \tau
$$

Multiplied by $\exp (-\omega t)$ for two sides of formula (9):

$$
\begin{gathered}
\|\boldsymbol{x}(t)\| \exp (-\omega t) \leq M a+\int_{0}^{t}\|M \exp (-\omega \tau) \Xi\| d \tau \\
\|\boldsymbol{x}(t)\| \exp (-\omega t) \leq M a+\int_{0}^{t}\|M \boldsymbol{x}(\tau) \exp (-\omega \tau) \Xi\| /\|\boldsymbol{x}(\tau)\| d \tau
\end{gathered}
$$

By Gronwall lemma,

$$
\|\boldsymbol{x}(t)\| \leq M a \exp \left[\int_{0}^{t}(\omega+\|M \Xi\| /\|\boldsymbol{x}(\tau)\|) d \tau\right]
$$

For simplification, we define as following:

$$
\vartheta(t)=\int_{0}^{t}(\omega+\|M \Xi\| /\|\boldsymbol{x}(\tau)\|) d \tau
$$

The system is stable for $\forall t \rightarrow+\infty, \lim _{t \rightarrow+\infty} \vartheta(t)<\varepsilon \mid<+\infty$ when there exists finite constant $|\varepsilon|<\infty$. There exists nonlinear semi-group $\pi_{t}$ such that state-space of system is described by following from formula (7):

From formula (12)-(13):

$$
\boldsymbol{x}(t)=\pi_{t} \boldsymbol{x}(0)
$$

$$
\|\boldsymbol{x}(t)\|=\left\|\pi_{t} \boldsymbol{x}(0)\right\| \leq \operatorname{Ma} \exp (\vartheta(t))
$$

Therefore, the nonlinear semi-group $\pi_{t}$ is stable when $\vartheta(t)<0$, in other words, the system (2)-(3) with external disturbances is stable. As a result, the system holds stable when the following condition is satisfied:

$$
\|\Xi\|<-\omega\|\boldsymbol{x}(\tau)\| / M
$$

Substitute the formula above into (8):

$$
\|\boldsymbol{B} \boldsymbol{u}(t)+\boldsymbol{h}(\boldsymbol{x}(t), \boldsymbol{u}(t))+\boldsymbol{g}(\boldsymbol{x}(t), \boldsymbol{u}(t), \boldsymbol{d}(t), t)\|<-\omega\|\boldsymbol{x}(\tau)\| / M
$$

And then

$$
\|\boldsymbol{B} \boldsymbol{u}(t)+\boldsymbol{h}(\boldsymbol{x}(t), \boldsymbol{u}(t))+\boldsymbol{g}(\boldsymbol{x}(t), \boldsymbol{u}(t), \boldsymbol{d}(t), t)\|>\|\boldsymbol{B} \boldsymbol{u}(t)\|-\|\boldsymbol{h}(\boldsymbol{x}(t), \boldsymbol{u}(t))\|+\|\boldsymbol{g}(\boldsymbol{x}(t), \boldsymbol{u}(t), \boldsymbol{d}(t), t)\|
$$

where, $\boldsymbol{u}(t)$ and $\boldsymbol{h}(\boldsymbol{x}(t), \boldsymbol{u}(t))$ are known. With definition $\beta_{0}(t)=\|\boldsymbol{B} \boldsymbol{u}(t)\|, \beta_{1}(t)=\|\boldsymbol{h}(\boldsymbol{x}(t), \boldsymbol{u}(t))\|$ Substitute into (16) and (17):

$$
\begin{array}{r}
\beta_{0}(t)-\beta_{1}(t)+\|\boldsymbol{g}(\boldsymbol{x}(t), \boldsymbol{u}(t), \boldsymbol{d}(t), t)\|<-\omega\|\boldsymbol{x}(\tau)\| / M \\
\|\boldsymbol{g}(\boldsymbol{x}(t), \boldsymbol{u}(t), \boldsymbol{d}(t), t)\|<\left(\beta_{1}(t)-\beta_{0}(t)\right)-\omega\|\boldsymbol{x}(\tau)\| / M
\end{array}
$$


The system (2)-(3) with external disturbances is stable when the constraint condition of external disturbances satisfies the form of (18). Furthermore, if there exist $\beta_{3} \in R^{+}$ make $\left(\beta_{1}(t)-\beta_{0}(t)\right) \leq \beta_{3}\|x(\tau)\|$, formula (18) can be rewritten as:

$$
\|\boldsymbol{g}(\boldsymbol{x}(t), \boldsymbol{u}(t), \boldsymbol{d}(t), t)\|<\left(\beta_{3}-\omega / M\right)\|\boldsymbol{x}(\tau)\|
$$

The constraint condition of external disturbances satisfies the inequation:

$$
\|\boldsymbol{g}(\boldsymbol{x}(t), \boldsymbol{u}(t), \boldsymbol{d}(t), t)\| /\|\boldsymbol{x}(\tau)\|<\beta_{3}-\omega / M
$$

where, the robustness performance index to external disturbances is defined as $\mathfrak{R}(\boldsymbol{A})=\omega / M$.

Remark 1: When the external disturbances constraint condition confines to (18), the missile pitching motion control system with external disturbances is stable. Obviously, not all of the external disturbances with the norm bounded assumption can make the system hold stable. In reference [10], authors assume the external disturbances are norm bounded simply, but whether the system can hold stable or not is not researched in-depth on this assumption. Just take the missile pitching control system (2)-(3) as an example. We denote the constraint condition of external disturbances as A2: $\lambda_{0}=\sup _{t \in[0, T]}\|\boldsymbol{g}(\boldsymbol{x}(t), \boldsymbol{u}(t), \boldsymbol{d}(t), t)\|$.

If $\lambda_{0} \geq\left(\beta_{1}(t)-\beta_{0}(t)\right)-\omega\|\boldsymbol{x}(\tau)\| / M$, system (2)-(3) are not stable on the norm bounded assumption. Therefore, it is meaningful to study the effect of the external disturbances on system stability in the design of control laws and fault diagnosis algorithm.

\section{Robust Fault Diagnosis Observer Design}

In this section, the robust fault diagnosis observer is proposed. And robustness performance index is defined to ensure the robustness of the observer. By linearization to nonlinear function $\boldsymbol{h}(\boldsymbol{x}(t), \boldsymbol{u}(t))$, nonlinear system (3) can be regarded as the linear system with uncertainty formula $\boldsymbol{g}(\boldsymbol{x}(t), \boldsymbol{u}(t), \boldsymbol{d}(t), t)$. Therefore, the gain matrix $\boldsymbol{G}$ of robust fault diagnosis can be designed by pole assignment based on traditional linear system theory.

Theorem 2: The robust fault diagnosis observer (19)-(20) of system (2)-(3) with fault $\beta_{2}=\sup _{t \in[0, T]}\|\boldsymbol{f}(t)\|$ and external disturbances is asymptotic convergence. Therefore, the robust performance index of observer satisfies the inequality as following:

$$
-\mathfrak{R}(\boldsymbol{A}-\boldsymbol{G C})>\lambda_{1}+\left(\|\boldsymbol{g}(\boldsymbol{x}(\tau), \boldsymbol{u}(\tau), \boldsymbol{d}(\tau), \tau)\|+\beta_{2}\right) /\|\boldsymbol{e}(\tau)\|
$$

Proof: Construct the robust fault diagnosis observer as following for the missile pitching motion control system (2)-(3) with fault and external disturbances:

$$
\begin{aligned}
& \dot{\hat{\boldsymbol{x}}}(t)=\boldsymbol{A} \hat{\boldsymbol{x}}(t)+\boldsymbol{B} \boldsymbol{u}(t)+\boldsymbol{h}(\hat{\boldsymbol{x}}(t), \boldsymbol{u}(t))+\boldsymbol{G}[\boldsymbol{y}(t)-\hat{\boldsymbol{y}}(t)] \\
& \hat{\boldsymbol{y}}(t)=\boldsymbol{C} \hat{\boldsymbol{x}}(t)
\end{aligned}
$$

The states estimation errors and residuals are defined as:

$$
\begin{aligned}
& \boldsymbol{e}(t)=\boldsymbol{x}(t)-\dot{\hat{\boldsymbol{x}}}(t) \\
& \boldsymbol{r}(t)=\boldsymbol{y}(t)-\hat{\boldsymbol{y}}(t)
\end{aligned}
$$

Substitute (3) and (19) into formula (21):

$$
\begin{aligned}
\dot{\boldsymbol{e}}(t) & =\dot{\boldsymbol{x}}(t)-\dot{\hat{\boldsymbol{x}}}(t) \\
& =\boldsymbol{A x}(t)-\boldsymbol{A} \hat{\boldsymbol{x}}(t)+\boldsymbol{h}(\boldsymbol{x}(t), \boldsymbol{u}(t))-\boldsymbol{h}(\hat{\boldsymbol{x}}(t), \boldsymbol{u}(t))-\boldsymbol{G}[\boldsymbol{C} \boldsymbol{x}(t)-\boldsymbol{C} \hat{\boldsymbol{x}}(t)]+\boldsymbol{g}(\boldsymbol{x}(t), \boldsymbol{u}(t), \boldsymbol{d}(t), t)+\boldsymbol{f}(t) \\
\dot{\boldsymbol{e}}(t) & =(\boldsymbol{A}-\boldsymbol{G C}) \boldsymbol{e}(t)+\boldsymbol{h}(\boldsymbol{x}(t), \boldsymbol{u}(t))-\boldsymbol{h}(\hat{\boldsymbol{x}}(t), \boldsymbol{u}(t))+\boldsymbol{g}(\boldsymbol{x}(t), \boldsymbol{u}(t), \boldsymbol{d}(t), t)+\boldsymbol{f}(t) \\
\boldsymbol{r}(t) & =\boldsymbol{y}(t)-\hat{\boldsymbol{y}}(t)=\boldsymbol{C} \boldsymbol{e}(t)
\end{aligned}
$$

The states estimation errors of system (23)-(24) are: $\boldsymbol{e}_{0}=\boldsymbol{e}(0), b=\|\boldsymbol{e}(0)\|$. with definition: $\Psi=\boldsymbol{h}(\boldsymbol{x}(\tau), \boldsymbol{u}(\tau))-\boldsymbol{h}(\hat{\boldsymbol{x}}(\tau), \boldsymbol{u}(\tau))+\boldsymbol{f}(t)+\boldsymbol{g}(\boldsymbol{x}(\tau), \boldsymbol{u}(\tau), \boldsymbol{d}(\tau), \tau)$. As a result, the states estimation errors are: 


$$
\boldsymbol{e}(t)=\exp [(\boldsymbol{A}-\boldsymbol{G} \boldsymbol{C}) t] \boldsymbol{e}(0)+\int_{0}^{t} \exp [(\boldsymbol{A}-\boldsymbol{G C})(t-\tau)] \Psi d \tau
$$

Apply the 2-norm to both sides of (25):

$$
\|\boldsymbol{e}(t)\|=\left\|\exp [(\boldsymbol{A}-\boldsymbol{G} \boldsymbol{C}) t] \boldsymbol{e}(0)+\int_{0}^{t} \exp [(\boldsymbol{A}-\boldsymbol{G} \boldsymbol{C})(t-\tau)] \Psi d \tau\right\|
$$

The system matrix $\boldsymbol{A}-\boldsymbol{G C}$ is Hurwitz when system (23)-(24) are stable, therefore, it can form an operator set for purpose of generating a stable linear semi-group $\zeta_{t}$.

Consequently, there exists $M \geq 1, \omega<0, t \geq 0$ such that $\left\|\zeta_{t}\right\| \leq M \exp (\omega t)$. Therefore, formula (26) fulfills the inequality as following:

$$
\|\boldsymbol{e}(t)\| \leq M b \exp (\omega t)+\int_{0}^{t}\|M \exp [\omega(t-\tau)] \Psi\| d \tau
$$

Multiplied by $\exp (-\omega t)$ for formula (27):

$$
\begin{gathered}
\|\boldsymbol{e}(t)\| \exp (-\omega t) \leq M b+\int_{0}^{t}\|M \exp (-\omega \tau) \Psi\| d \tau \\
\|\boldsymbol{e}(t)\| \exp (-\omega t) \leq M b+\int_{0}^{t}\|M \boldsymbol{e}(\tau) \exp (-\omega \tau) \Psi\| /\|\boldsymbol{e}(\tau)\| d \tau
\end{gathered}
$$

Not loss of generality, we assume the fault injected into the missile pitching motion control system is: $\beta_{2}=\sup _{t \in[0, T]}\|\boldsymbol{f}(t)\|$

$$
\begin{gathered}
\|\Psi\| \leq\|\boldsymbol{h}(\boldsymbol{x}(\tau), \boldsymbol{u}(\tau))-\boldsymbol{h}(\hat{\boldsymbol{x}}(\tau), \boldsymbol{u}(\tau))\|+\|\boldsymbol{f}(t)\|+\|\boldsymbol{g}(\boldsymbol{x}(\tau), \boldsymbol{u}(\tau), \boldsymbol{d}(\tau), \tau)\| \\
\leq \lambda_{1}\|\boldsymbol{e}(\tau)\|+\beta_{2}+\|\boldsymbol{g}(\boldsymbol{x}(\tau), \boldsymbol{u}(\tau), \boldsymbol{d}(\tau), \tau)\| \\
\|\boldsymbol{e}(t)\| \leq M b \exp \left\{\int_{0}^{t}[\omega+M\|\Psi\| /\|\boldsymbol{e}(\tau)\| \mid d \tau\}\right.
\end{gathered}
$$

Consequently, the fault diagnosis observer (19)-(20) is asymptotic convergence when the following formula holds:

$$
\begin{gathered}
\omega+M\|\Psi\| /\|\boldsymbol{e}(\tau)\|<0 \\
\left.-\omega / M>\lambda_{1}+(\|\boldsymbol{g}(\boldsymbol{x}(\tau), \boldsymbol{u}(\tau), \boldsymbol{d}(\tau), \tau)\|)+\beta_{2}\right) /\|\boldsymbol{e}(\tau)\|
\end{gathered}
$$

The robustness performance index $\mathfrak{R}(\boldsymbol{A}-\boldsymbol{G C})$ of observer (19)-(20) satisfies the following constraint condition from section 2.1:

$$
-\mathfrak{R}(\boldsymbol{A}-\boldsymbol{G C})>\lambda_{1}+\left(\|\boldsymbol{g}(\boldsymbol{x}(\tau), \boldsymbol{u}(\tau), \boldsymbol{d}(\tau), \tau)\|+\beta_{2}\right) /\|\boldsymbol{e}(\tau)\|
$$

Consequently, the fault diagnosis observer is asymptotic convergence when formula (32) holds. And then, the gain matrix of fault diagnosis observer can be solved by robust performance index proposed.

Remark 2: The maximum tolerant values of states estimation errors $\left\|\boldsymbol{e}_{\max }(\tau)\right\|$ are known a prior in the process of missile attitude control system and the supreme values $\left\|\boldsymbol{g}_{\max }(\boldsymbol{x}(\tau), \boldsymbol{u}(\tau), \boldsymbol{d}(\tau), \tau)\right\|$ can be obtained by Theorem 1.

Denote: $\mathfrak{R}(\boldsymbol{A}-\boldsymbol{G C}) \square \frac{1}{2} \lambda_{\max }\left[(\boldsymbol{A}-\boldsymbol{G C})+(\boldsymbol{A}-\boldsymbol{G C})^{T}\right]$. Substitute it into (32):

$$
\lambda_{i}\left[(\boldsymbol{A}-\boldsymbol{G} \boldsymbol{C})+(\boldsymbol{A}-\boldsymbol{G} \boldsymbol{C})^{T}\right]<-2\left\{\lambda_{1}+\left(\|\boldsymbol{g}(\boldsymbol{x}(\tau), \boldsymbol{u}(\tau), \boldsymbol{d}(\tau), \tau)\|+\beta_{2}\right) /\|\boldsymbol{e}(\tau)\|\right\}
$$

where, $\lambda_{\max }(\cdot)$ and $\lambda_{i}(\cdot)$ represent the maximum and arbitrary eigenvalue for matrix $(\cdot)$, the gain matrix $\boldsymbol{G}$ of fault diagnosis observer can be solved by pole assignment when the robust performance index $\mathfrak{R}(\boldsymbol{A}-\boldsymbol{G C})$ is given.

\section{Fault Diagnosis Algorithm and Adaptive Threshold Design}

Fault diagnosis algorithm involves residual generation, residual processing and decision-making as depicted in Figure 2. Usually, compared the residuals with threshold to diagnose fault: 


$$
\left\{\begin{array}{l}
\|\boldsymbol{r}(t)\|_{2, T}>\boldsymbol{J}_{\mathrm{th}}(\boldsymbol{r}(t)) \Rightarrow \text { fault } \\
\|\boldsymbol{r}(t)\|_{2, T} \leq \boldsymbol{J}_{\mathrm{th}}(\boldsymbol{r}(t)) \Rightarrow \text { fault }- \text { free }
\end{array}\right.
$$

The residuals are obtained from (24) in the fault-free mode: $\boldsymbol{r}(t)=\boldsymbol{C e}(t)$.

The states estimation errors are:

$$
\boldsymbol{e}(t)=\boldsymbol{e}(0)+\int_{0}^{t}[\boldsymbol{h}(\boldsymbol{x}(\tau), \boldsymbol{u}(\tau))-\boldsymbol{h}(\hat{\boldsymbol{x}}(\tau), \boldsymbol{u}(\tau))+\boldsymbol{g}(\boldsymbol{x}(\tau), \boldsymbol{u}(\tau), \boldsymbol{d}(\tau), \tau)] d \tau
$$

Therefore,

$$
\boldsymbol{r}(t)=\boldsymbol{C e}(0)+\boldsymbol{C} \int_{0}^{t}[\boldsymbol{h}(\boldsymbol{x}(\tau), \boldsymbol{u}(\tau))-\boldsymbol{h}(\hat{\boldsymbol{x}}(\tau), \boldsymbol{u}(\tau))+\boldsymbol{g}(\boldsymbol{x}(\tau), \boldsymbol{u}(\tau), \boldsymbol{d}(\tau), \tau)] d \tau
$$

Apply the 2-norm for formula (35):

$$
\|\boldsymbol{r}(t)\|=\left\|\boldsymbol{C e}(0)+\boldsymbol{C} \int_{0}^{t}[\boldsymbol{h}(\boldsymbol{x}(\tau), \boldsymbol{u}(\tau))-\boldsymbol{h}(\hat{\boldsymbol{x}}(\tau), \boldsymbol{u}(\tau))+\boldsymbol{g}(\boldsymbol{x}(\tau), \boldsymbol{u}(\tau), \boldsymbol{d}(\tau), \tau)] d \tau\right\|
$$

with definition: $b_{c}=\|\boldsymbol{C}\|$ and

$$
\delta(\boldsymbol{h})=\boldsymbol{h}(\boldsymbol{x}(\tau), \boldsymbol{u}(\tau))-\boldsymbol{h}(\hat{\boldsymbol{x}}(\tau), \boldsymbol{u}(\tau))
$$

Therefore,

$$
\|\boldsymbol{r}(t)\| \leq b b_{c}+b_{c}\left\|\int_{0}^{t}[\delta(\boldsymbol{h})+\boldsymbol{g}(\boldsymbol{x}(\tau), \boldsymbol{u}(\tau), \boldsymbol{d}(\tau), \tau)] d \tau\right\|
$$

Formula (36) can be rewritten as:

$$
\|\delta(\boldsymbol{h})\| \leq \lambda_{1}\|\boldsymbol{x}(\tau)-\hat{\boldsymbol{x}}(\tau)\|=\lambda_{1}\|\boldsymbol{e}(\tau)\|=\frac{\lambda_{1}}{b_{c}}\|\boldsymbol{r}(\tau)\|
$$

As a result,

$$
\|\boldsymbol{r}(t)\| \leq b b_{c}+\int_{0}^{t}\left[\lambda_{1}\|\boldsymbol{r}(\tau)\|+b_{c}\|\boldsymbol{g}(\boldsymbol{x}(\tau), \boldsymbol{u}(\tau), \boldsymbol{d}(\tau), \tau)\|\right] d \tau
$$

Formula (39) can be further rewritten as:

$$
\|\boldsymbol{r}(t)\| \leq b b_{c}+\int_{0}^{t} \lambda_{1} b_{c}\|\boldsymbol{e}(\tau)\| d \tau+\int_{0}^{t} b_{c}\left[\beta_{1}(\tau)-\beta_{0}(\tau)-\omega\|\boldsymbol{x}(\tau)\| / M\right] d \tau
$$

where, maximum tolerant values of states estimation errors $e_{\max }$ and the system state stable value $x_{\text {sta }}$ are known.

It can be obtained from (40)

$$
\left\{\begin{array}{l}
e_{\max }=\left\|\boldsymbol{e}_{\max }(t)\right\| \\
\lim _{t \rightarrow T}\|\boldsymbol{x}(t)\| \rightarrow x_{\text {sta }}
\end{array}\right.
$$

$$
\|\boldsymbol{r}(t)\| \leq b b_{c}+\lambda_{1} b_{c} e_{\max } t+b_{c} \int_{0}^{t}\left[\beta_{1}(\tau)-\beta_{0}(\tau)-\omega x_{\max } / M\right] d \tau
$$

When the system is fault case:

$$
\|\boldsymbol{r}(t)\|>b b_{c}+\lambda_{1} b_{c} e_{\max } t+b_{c} \int_{0}^{t}\left[\beta_{1}(\tau)-\beta_{0}(\tau)-\omega x_{\max } / M\right] d \tau
$$

As a result, the adaptive threshold of the fault diagnosis observer designed is:

$$
\boldsymbol{J}_{\mathrm{th}}(\boldsymbol{r}(t))=b b_{c}+\left(\lambda_{1} b_{c} e_{\max }-\omega x_{\max } / M\right) t+b_{c} \int_{0}^{t}\left[\beta_{1}(\tau)-\beta_{0}(\tau)\right] d \tau
$$

The threshold is sufficiently considered the external disturbances. Threshold derived is adaptive. Compared with the traditional methods on assumption that the threshold is constant, the fault diagnosis algorithm with adaptive threshold proposed decreases false alarm rates in diagnosis process.

\section{Simulation}

The simulations are performed on the missile pitching control system in order to verify the effectiveness of the fault diagnosis algorithm proposed. It is necessary to notice that aerodynamic parameters are given by quadratic polynomial fitting because of the private aspect about missile parameters. 


\subsection{Simulation Parameters}

The differential equations of missile pitching motion control system are represented as following [6]:

$$
\left\{\begin{array}{l}
m \frac{d v}{d t}=p \cos \alpha-X(m a, \alpha)-m g \sin \theta \\
m v \frac{d \theta}{d t}=p \sin \alpha+Y(m a, \alpha)-m g \cos \theta \\
J_{z} \frac{d \omega_{z}}{d t}=M_{z} \\
\frac{d x}{d t}=v \cos \theta \\
\frac{d y}{d t}=v \sin \theta \\
\frac{d \vartheta}{d t}=\omega_{z} \\
\alpha=\vartheta-\theta
\end{array}\right.
$$

where, the related parameters are in reference [6] and then some explanations are given. The pitching moment: $M_{z} \approx \varphi_{1}(m a, \alpha)+\varphi_{2}(m a, \alpha) \delta_{z}+\varphi_{3}\left(\alpha, \omega_{z}, v\right) . \delta_{z}$ is the evaluator angle of missile pitching control system, and the pitching moment is negative when the angle is positive-skewed, whereas negative-skewed. The missile aerodynamic parameters are as following: $X(m a, \alpha), Y(m a, \alpha), \varphi_{1}(m a, \alpha), \varphi_{2}(m a, \alpha), \varphi_{3}\left(\alpha, \omega_{z}, v\right)$.The resistance and lifting are depicted in Fig.3-4, the force situation of missile with different flight states is diverse each other from Fig.3-4.

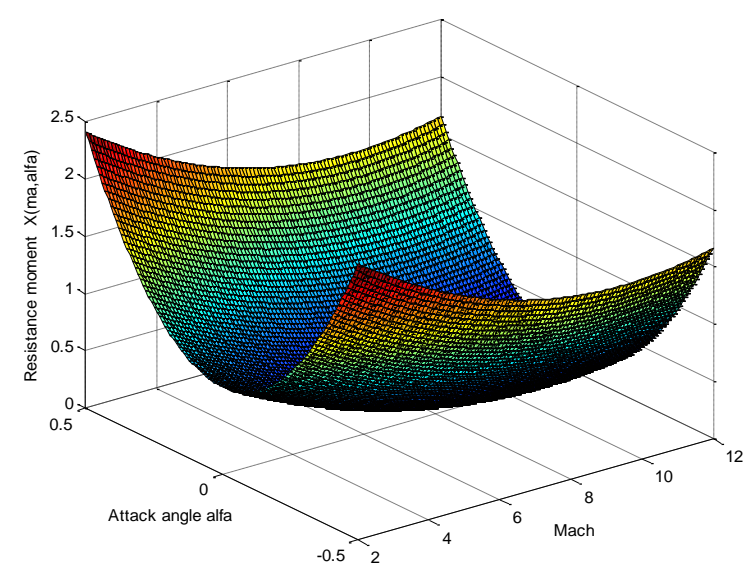

Figure 3. The Missile Resistance 


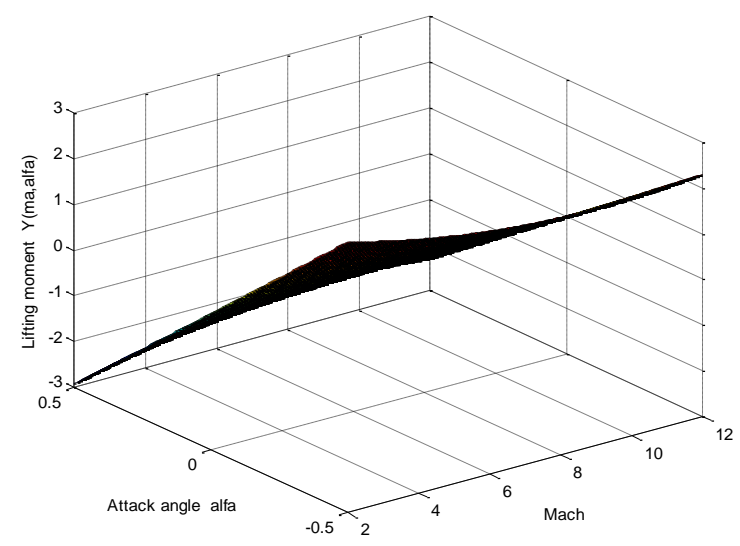

Figure 4. The Missile Lifting

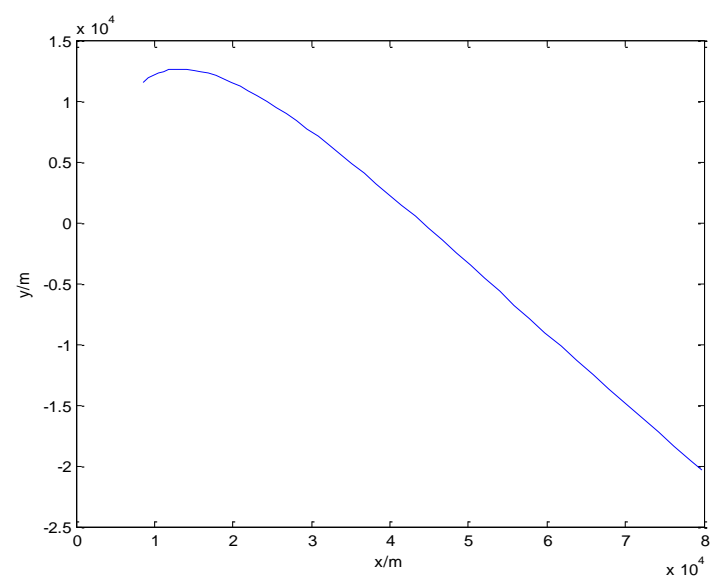

Figure 5. The Missile Trajectory Character

The parameters of missile pitching moment control system in the atmosphere are:

The missile empty weight is $230 \mathrm{~kg}$, Z-axis rotational inertia is $J_{z}=247.26 \mathrm{~kg} \cdot \mathrm{m}^{2}$, generator impulse thrust of missile attitude control system is $p=2200 \mathrm{~N}$. The initial location of missile in inertial coordinates system is $x_{0}=8530 \mathrm{~m}, y=11600 \mathrm{~m}$; missile initial velocity is $v=300 \mathrm{~m} / \mathrm{s}$, and trajectory pitching angle is $\theta=0.536 \mathrm{rad}$. The missile trajectory characters are depicted in Fig.5.

$$
\begin{aligned}
\boldsymbol{A}_{0} & =\left[\begin{array}{ccccccc}
0 & 0 & 0 & 0 & 0 & 0 & 0 \\
0 & 0 & 0 & 0 & 0 & 0 & 0 \\
0 & 0 & 0 & 0 & 0 & 0 & 0 \\
0 & 0 & 0 & 0 & 0 & 0 & 0 \\
0 & 0 & 0 & 0 & 0 & 0 & 0 \\
0 & 0 & 1 & 0 & 0 & 0 & 0 \\
0 & -1 & 0 & 0 & 0 & 1 & 0
\end{array}\right], \Delta \boldsymbol{A}=\boldsymbol{E}_{1} \Sigma_{1} \boldsymbol{F}_{1} \quad \text {, and } \quad \boldsymbol{A}=\boldsymbol{A}_{0}+\Delta \boldsymbol{A} \\
\boldsymbol{B} & =0_{7 \times 1}, \boldsymbol{E}_{1}=\boldsymbol{F}_{1}=\boldsymbol{I}, \boldsymbol{\Sigma}_{1}=0.5 \boldsymbol{I} .
\end{aligned}
$$




$$
\boldsymbol{g}(\boldsymbol{x}(t), u(t), \boldsymbol{d}(t))=\left[\begin{array}{c}
p \cos \alpha-X(m a, \alpha)-m g \sin \theta+d_{1}(t) \\
\frac{1}{m v}[p \sin \alpha+Y(m a, \alpha)-m g \cos \theta]+d_{2}(t) \\
\frac{1}{J_{z}}\left[\varphi_{1}(m a, \alpha)+\varphi_{2}(m a, \alpha) \delta_{z}+\varphi_{3}\left(\alpha, \omega_{z}, v\right)\right]+d_{3}(t) \\
v \cos \theta+d_{4}(t) \\
v \sin \theta+d_{5}(t) \\
d_{6}(t) \\
d_{7}(t)
\end{array}\right]
$$

The system disturbances are: $d_{i}(t)=0.01 \sin (t) i=1,2 \ldots 7$. Obviously, it is not suitable for missile control system design on the assumption that the external disturbances are defined as norm bounded in the process of the missile pitching control system design. The constraint condition of the external disturbances can be derived by the section 3 .

\subsection{Performance Analysis for the Fault Diagnosis Algorithm}

The supreme of missile external disturbances in the consider period under the Simulink condition from Theorem 1 is: $\|\boldsymbol{g}\|_{\max }=9.69507 \times 10^{5}$. Therefore, not all of the external disturbances can satisfy hypothetical norm bounded form and the prior constraint condition on external disturbances restricts the generality of fault diagnosis algorithm even the stability of the control system. The maximum tolerant value of states estimation errors are defined as:

$$
\boldsymbol{e}_{\max }=\left[\begin{array}{lllllll}
40 & 0.01 & 2 & 20 & 20 & 0.02 & 0.02
\end{array}\right]
$$

Therefore, $\|\boldsymbol{e}\|_{\max }=1.202 \times 10^{3}$. From theorem 2 and remark 2:

$$
\lambda_{i}\left[(\boldsymbol{A}-\boldsymbol{G} \boldsymbol{C})+(\boldsymbol{A}-\boldsymbol{G C})^{T}\right]<-806.5786
$$

The fault diagnosis observer designed is asymptotic convergence robust fault diagnosis observer when the poles are placed at the left plane of -806.5786 . It should be noticed that the gain matrix $G$ is not unique because of the poles selected, in this paper, the poles are settled on:

$$
p=[-850+5 \mathrm{i},-850-5 \mathrm{i},-880+6 \mathrm{i},-880-6 \mathrm{i},-900+10 \mathrm{i},-900-10 \mathrm{i},-1000]
$$

The gain matrix $\boldsymbol{G}$ of the observer proposed can be obtained by the pole assignment:

$$
\boldsymbol{G}=\left[\begin{array}{ccccccc}
850 & 5 & 0 & 0 & 0 & 0 & 0 \\
-5 & 850 & 0 & 0 & 0 & 0 & -1 \\
0 & 0 & 880 & 6 & 0 & 1 & 0 \\
0 & 0 & -6 & 880 & 0 & 0 & 0 \\
0 & 0 & 0 & 0 & 900 & 10 & 0 \\
0 & 0 & 0 & 0 & -10 & 900 & 1 \\
0 & 0 & 0 & 0 & 0 & 0 & 1000
\end{array}\right]
$$

As a result, substitute the gain matrix $\boldsymbol{G}$ into the observer (23)-(24) and then the residual effect is depicted in Fig.6 by Simulink. Not loss of generality, just take the 3rd channel residual of fault diagnosis as an example to research and assume the missile attitude control system with the fault as following:

$$
f= \begin{cases}0.2 & 60 s>t \geq 50 s \\ 0 & t=\text { other }\end{cases}
$$




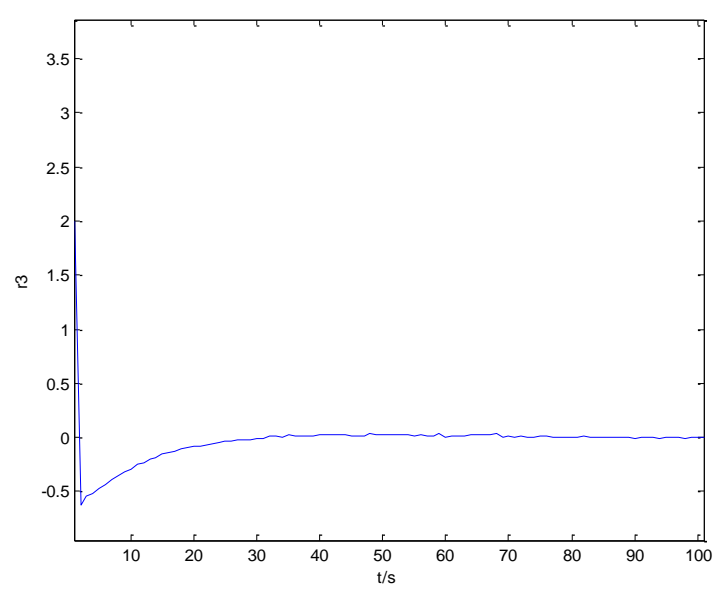

Figure 6. The 3rd Channel Residual of Fault Diagnosis Observer

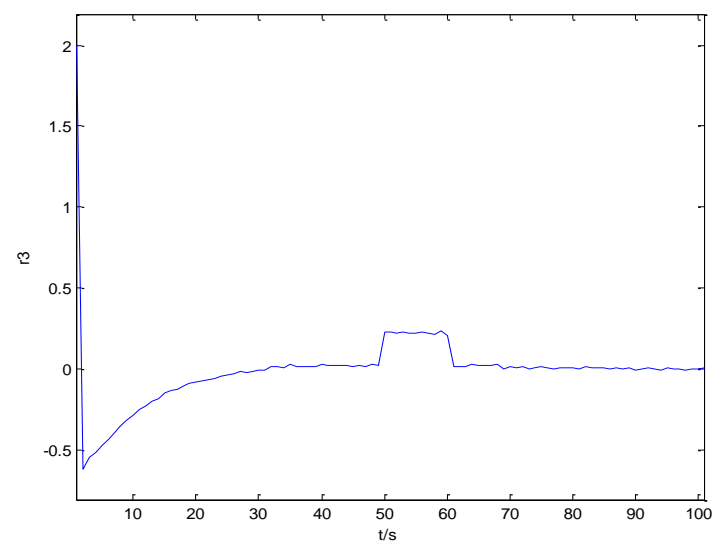

Figure 7. Result of Fault Diagnosis

The residual of the fault diagnosis is asymptotic convergence and has the robustness to the external disturbances from Figure 6. The good convergence of residual illustrates that the robust fault diagnosis observer proposed in theorem 2 is effectiveness. Furthermore, the smooth residual curve also illustrates that the external disturbances constraint condition which satisfies the system stable is reasonable and the defined robust performance index is practicable.

The Figure 7 demonstrates the effectiveness of the fault diagnosis algorithm to the fault and the system fault character is revealed in Figure 7. We can conclude whether the fault occurs or not by comparing the threshold designed. From the simulation results and the analysis above we can conclude that the robust fault diagnosis algorithm proposed for the missile pitching motion control system is effectiveness.

\section{Conclusion}

In this paper, the disturbances constraint condition that satisfies the system stability is derived to improve the limitation of external disturbances is norm bounded in most of the nonlinear system fault diagnosis scheme. And then, robustness performance index is defined to ensure the robustness of the fault diagnosis observer designed. In the decisionmaking unit, adaptive threshold is designed. Finally, the simulations are performed on the missile attitude control system. Simulation results show the effectiveness of the algorithm proposed. 


\section{References}

[1] Design and flight test of a robust autopilot for the IRIS-T air-to-air missile, Control Engineering Practice, vol. 11, no. 5, (2003), pp. 551-558.

[2] J. Harris and N. Slegers, "Performance of a fire-and-forget anti-tank missile with a damaged wing", Mathematical and Computer Modeling, vol. 50, nos. 1-2, (2009), pp. 292-305.

[3] S. Chaib, D. Boutat, A. Banali and F. Kratz, "Invertibility of Switched Nonlinear Systems Application to Missile Faults Reconstruction”, In: Proceedings of the 46th IEEE Conference on Decision and Control. New Orleans, LA, USA: IEEE, (2007), pp. 12-14.

[4] M. A. Wael and Q. Quan, "Robust Hybrid Control for Ballistic Missile Longitudinal Autopilot", Chinese Journal of Aeronautics, vol. 24, no. 6, (2011), pp. 777-788.

[5] A. Tsourdos and B. A White, "Adaptive flight control design for nonlinear missile", Control Engineering Practice, vol. 13, no. 3, (2005), pp. 373-382.

[6] G M. Siouris, "Missile guidance and control systems", New York: Springer-Verlag, (2004).

[7] J. Zarei and J. Poshtan, "Sensor fault detection and diagnosis of a process using unknown input observer", Mathematical and Computational Applications, vol. 16, no. 1, (2011), pp. 31-42.

[8] C. Hajiyev and F. Caliskan, "Sensor and control surface/actuator failure detection and isolation applied to F-16 flight dynamic", Aircraft Engineering and Aerospace Technology, 2005, 77(2): 152-160.

[9] H R Karimi, M Zapateiro, N Luo. A linear matrix inequality approach to robust fault detection filter design of linear systems with mixed time-varying delays and nonlinear perturbations. Journal of the Franklin Institute, 2010, 347(6): 957-973.

[10] Leishi Bai, Zuohua Tian, Songjiao Shi. Robust fault detection for a class of nonlinear time-delay systems. Journal of the Franklin Institute, 2007, 344(6): 873-888.

[11] Prashant Mhaskar, Charles McFall, Adiwinata Gani, Panagiotis D Christofides, James F Davis. Isolation and handling of actuator faults in nonlinear systems. Automatica, 2008, 44(1): 53-62.

[12] Juan C Cruz-Victoria, Rafael Martınez-Guerra, Jose Juan Rincon-Pasaye. On nonlinear systems diagnosis using differential and algebraic methods. Journal of the Franklin Institute, 2008, 345(2): 102118 .

[13] N Barhoumi, F Msahli, M Djemaï, K Busawon. Observer design for some classes of uniformly observable nonlinear hybrid systems. Nonlinear Analysis: Hybrid Systems, 2012, 6(4): 917-929.

[14] Jie Yu. A nonlinear kernel Gaussian mixture model based inferential monitoring approach for fault detection and diagnosis of chemical processes. Chemical Engineering Science, 2012, 68(1): 506-519.

[15] Dimitrios Fragkoulisa, Gilles Roux, Boutaieb Dahhou. Detection, isolation and identification of multiple actuator and sensor faults in nonlinear dynamic systems: Application to a waste water treatment process. Applied Mathematical Modeling, 2011, 35(1): 522-543.

[16] Ming Fang, Yantao Tian, Li Guo. Fault diagnosis of nonlinear system based on generalized observer. Applied Mathematics and Computation, 2007, 185(2): 1131-1137.

[17] Guan-Chun Luh, Wei-Chong Cheng. Immune model-based fault diagnosis. Mathematics and Computers in Simulation, 2005, 67(6): 515-539.

[18] A Pazy. Semi-groups of Linear Operators and Applications to Partial Differential Equations. New York: Springer-Verlag, 1983.

[19] P A Ioannou, J Sun. Robust Adaptive Control. New York: Prentice-Hall, 1996.

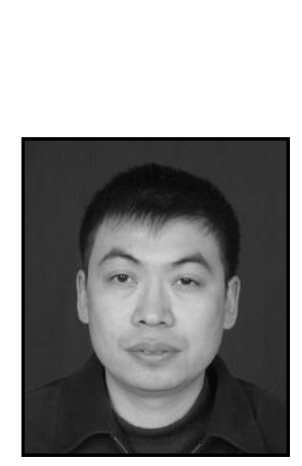

\section{Authors}

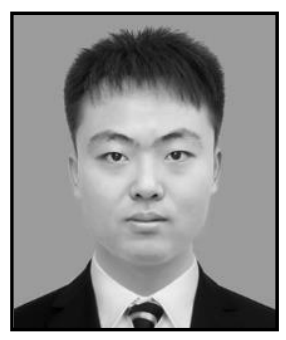

Zhao Jing-yun, Associate Professor at Department of Mechanical Engineering, Henan Mechanical and Electrical Engineering, his research interest fields include mechanical design and manufacture.

Fu Zhen-duo, He received the Ph.D. degree from College of Automation, Harbin Engineering University. His research interests include fault diagnosis and fault tolerant control technology and its applications. $\mathrm{He}$ is the corresponding author this paper. E-mail: fuzhenduo@126.com 